\title{
Review of: "Manufacturing process of short carbon fiber reinforced Al matrix with preformless and their properties"
}

\author{
Xiaofei YAN
}

Potential competing interests: The author(s) declared that no potential competing interests exist.

This manuscript mainly investigates the manufacturing process of short carbon fiber reinforced Al matrix with preformless and their properties. It is well-structured and good written. The following issues should be solved before publication:

1. Figure $2 \mathrm{~b}$ illustrates that a perfect, uniform Cu-plated layer was acquired over the SCFs after plating for $30 \mathrm{~s}$ and the density of the Cu-plated SCF is $2.24 \mathrm{~g} / \mathrm{cm}^{3}$ पhow about the Cu-plated layer on SCFs after plating for 30s-60s and below 30s?

2. The maximum infiltration pressure which the authors applied is only $0.8 \mathrm{MPa}$, how can you regard $0.8 \mathrm{MPa}$ is the most suitable pressure for fabricating composites? How about the infiltration pressure is over $0.8 \mathrm{MPa}$ ?

3. Please conform the units of the density? There must exit the errors. 